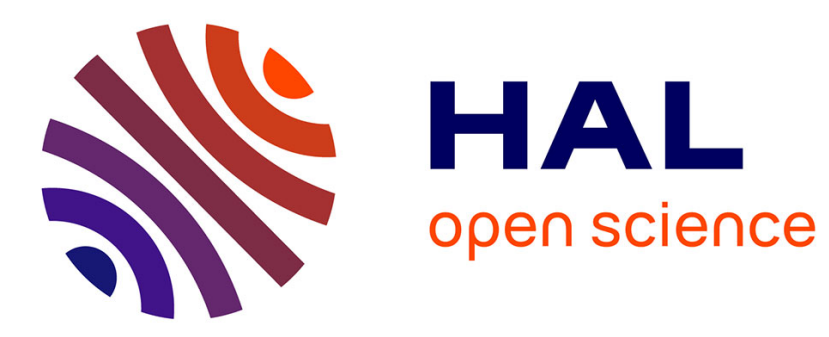

\title{
Dissemination strategies in realistic V2V highway networks: The Madrid trace case
}

Alexandre Mouradian, Veronique Veque

\section{To cite this version:}

Alexandre Mouradian, Veronique Veque. Dissemination strategies in realistic V2V highway networks: The Madrid trace case. Wireless Days (WD), 2016, Mar 2016, Toulouse, France. pp.1 3, 10.1109/WD.2016.7461486 . hal-01398516

\section{HAL Id: hal-01398516 https://hal.science/hal-01398516}

Submitted on 17 Nov 2016

HAL is a multi-disciplinary open access archive for the deposit and dissemination of scientific research documents, whether they are published or not. The documents may come from teaching and research institutions in France or abroad, or from public or private research centers.
L'archive ouverte pluridisciplinaire HAL, est destinée au dépôt et à la diffusion de documents scientifiques de niveau recherche, publiés ou non, émanant des établissements d'enseignement et de recherche français ou étrangers, des laboratoires publics ou privés. 


\title{
Dissemination Strategies in Realistic V2V Highway Networks: the Madrid Trace Case
}

\author{
Alexandre Mouradian and Véronique Vèque \\ Laboratoire des Signaux et Systèmes (L2S, UMR8506), \\ Université Paris Sud-CNRS-CentraleSupélec, \\ F-91192 Gif-sur-Yvette \\ firstname.lastname@u-psud.fr
}

\begin{abstract}
Multi-hop message dissemination in vehicular networks has been largely studied in the past decade. It notably allows to push information to the drivers concerning hazards that they will encounter ahead on the road such as, for instance, traffic jams, ice patches, etc. It could thus reduce the number of car crashes. During the dissemination of the information, each node have to decide if it will forward a received packet or not. In the literature, many forwarding strategies have been proposed, but they are usually evaluated by simulation with simple mobility models. In this paper, we investigate the performance of several dissemination schemes on the Madrid Highway vehicular mobility trace. This trace is a realistic micro-mobility model representing $10 \mathrm{~km}$ portions of two highways around the city of Madrid. The main result of this paper is to show that a carry-and-forward version of farthest node dissemination strategy is a very promising scheme under realistic highway traffic conditions, because this scheme increases the number of receivers and distance covered by the packet at a very low cost in terms of traffic overhead and algorithmic complexity.
\end{abstract}

Keywords-VANETs, Dissemination, Performance Evaluation

\section{INTRODUCTION}

Vehicular communications are an important part of Intelligent Transport Systems (ITS) which are now in their standardization phase. Among others, ETSI and ISO have defined a basic set of distributed applications [1] to be provided by intelligent vehicles and management systems. They have several targets: Public Safety, Traffic Efficiency Management, Traveler Information Support and Comfort of driver and passengers but also, Air pollution emission measurement and reduction, Electric Vehicle Itinerary Planning, Law enforcement and Broadband services. These applications can be distinguished according to their communication type either Vehicle-to-Vehicle (V2V) or Vehicle-to-Infrastructure (V2I), their Quality of Service (QoS), and their delivery requirements such as broadcasting, unicasting or geocasting. In this work, we focus on applications which rely on $\mathrm{V} 2 \mathrm{~V}$ and information dissemination. Active road safety applications, cooperative traffic efficiency and some cooperative local services fall in this category. We are especially interested in the dissemination of Decentralized Environmental Notification Message (DENM), as defined in [1], in order to alert road users of hazardous events in an Area of Interest. For these types of applications, simple link-level broadcasting is not enough, but multi-hop dissemination is needed. With multi-hop dissemination, the source of the information broadcasts a message to its neighbors, each node receiving the message decides to forward it in its vicinity or not according to the dissemination algorithm.
The process should stop when the message reaches the limit of the Area of Interest. In this paper, we focus on the different forwarding or relaying strategies proposed in the literature. A forwarding strategy is defined as the actions taken by a node when it receives a dissemination message. The simplest flooding algorithm which allows each node to relay a received packet to all its neighbors provoke the broadcast storm problem [2]. Many criteria have thus been proposed in the literature in order to reduce the number of packet copies and thus the contention in the network. Dissemination algorithms have been largely studied in the literature [2] [3] [4], but most of the time the studies are limited to simulations with low fidelity traffic models. In this work, in order to evaluate dissemination strategies, we use a realistic micro-mobility trace: the Madrid Highway trace [5].

The main forwarding strategies proposed in the literature are introduced in sections II and III. Then we provide the simulation setup in Section IV. Using the Madrid Highway trace, we focus on the simulation of generic forwarding strategies instead of their various implementations. In Section $\mathrm{V}$, we discuss the simulation results. We detail the global performance of the schemes in terms of number of receptions, traveled distance, replication and contention of the disseminated message. We also look at the timing dynamics of the schemes in order to evaluate their timeliness and thus their ability to be employed for road safety applications. Section VI concludes and provides future works.

\section{RELATED WORK}

Message dissemination in vehicular networks has been a subject of great interest in the past decade. Dissemination schemes are envisioned to be part of Intelligent Transport Systems (ITS) in order to push various information about the road network to the drivers [6]. In this paper we focus on multi-hop dissemination and especially forwarding strategies. The numerous proposed protocols can be classified into the following categories according to how the forwarding decision is made: location-based [7] [8] [9] [10] [4], probability-based [7] [11] [12] [13] [9], density-based [14]. These strategies are detailed in Section III.

Even if performance studies have been provided in order to evaluate these dissemination schemes [2] [4], they do not focus on the dissemination strategy principles but rather on specific implementations. Moreover the evaluation is performed on mobility traces which are built from scratch for the simulation purpose. For instance, the authors of [4] use a highway and a 
grid scenario where the mobility pattern of the vehicle is not detailed. In [2], the authors also use a highway scenario with vehicle traffic generated from a simulation tool according to well-known vehicular traffic patterns (free flow, car following, jam), but not from real data. In this work we go a step further by considering a realistic highway mobility pattern constructed from real world data [5] while evaluating generic dissemination strategies.

\section{DISSEMINATION ALGORITHMS}

In this section, we present the schemes which are evaluated in the remainder of the paper. These generic dissemination strategies: Blind Flooding, Probabilistic Flooding, Densitybased Flooding, Farthest node Flooding, Probabilistic Farthest node Flooding and carry-and-forward are taken from various sources of the literature such as [2] and [3] for instance. Nevertheless, in this paper, we do not investigate the performance of cluster-based schemes because, as shown in [2] this kind of dissemination algorithms does not perform very well for vehicle densities above 40 vehicle $/ \mathrm{km}$. In the Madrid trace we are seldom under this density [5].

The behavior of a dissemination scheme is defined by the actions taken upon a packet reception. In the remainder of this section, we thus describe these actions for all the considered schemes.

\section{A. Blind Flooding}

Blind flooding $(\mathrm{BF})$ is the simplest scheme: upon reception, a node checks if it has already received this packet. If not, it broadcasts it. In the other case, it silently discards it. BF is the most reliable scheme since the message is broadcasted by all the nodes. Nevertheless, it induces a huge amount of redundancy. As messages are transmitted multiple times in the same areas of the network, it causes congestion and collisions which can delay some message deliveries. Nonetheless, BF is a reference dissemination scheme to which others can be compared. The only information needed in the packet is an ID so that the nodes can decide if they have already seen the packet or not.

\section{B. Probabilistic Flooding}

For Probabilistic Flooding (PF), the nodes act the same as for $\mathrm{BF}$ except that they forward the packet (only if it is the first time it is received) with probability $p$ and discard it with probability $1-p$. Depending on the value of $p$, this scheme can drastically reduce the number of redundant transmissions. Nevertheless, there is no guaranty that the network is fully flooded on convergence of the algorithm. Once again, the only information needed in the packet is an ID.

\section{Density-based Flooding}

With Density-based Flooding (DF), the selection of the forwarder is based on the degree of the nodes. The packet is forwarded by the node with the highest degree in the neighborhood of the sender. It can be implemented in two ways: (1) neighbor nodes exchange their degrees using hello messages and the dissemination message is sent only to the neighbor with the highest degree, or (2) the message is broadcasted, the receivers initialize a timer which is inversely proportional to their degrees and the first node whose timer expires forwards the packet. The idea behind this scheme is that it is better to use the more connected nodes as forwarders since they will disseminate information to more nodes. We will see that this scheme does not perform very well in practice, at least in the Madrid trace case.

\section{Farthest node Flooding}

The Farthest node Flooding (FF) scheme is similar to the density-based in the sense that only one node is selected as a forwarder: the farthest from the sender. In this case as well, the two previously mentioned implementations are possible (for the timer-based implementation, the timer is function of the distance from the sender). Choosing the farthest node to forward the packet allows to cover rapidly a long distance in the flooding direction while keeping the traffic low. It is considered as the best dissemination scheme available [2] as will be confirmed in the case of the Madrid trace in Section V. To implement this scheme, the nodes have to know their position and direction (which is a standard requirement in VANETs [6]).

\section{E. Probabilistic Farthest node Flooding}

Probabilistic Farthest node Flooding (PFF) is a mix between the probabilistic and farthest node scheme. In this configuration, the nodes compute their probability of retransmitting the packet according to their distance to the sender. The farther from the emitter the node is, the higher the probability to retransmit.

\section{F. Carry-and-Forward Flooding}

The basic carry-and-forward scheme [15] [16] consists in keeping the packet in memory when no neighbors are available and forwarding it as soon as it is possible (when a neighbor is available). It aims at solving the partitioned network problem that can appear in case of low vehicular traffic. It can be adapted to any of the previously described forwarding policies. In this paper, we propose to adapt this scheme to FF: a node with no farther neighbor in the flooding direction has to wait until one appears and then it can forward the packet.

For all these schemes, providing that the nodes know their locations and directions, the flooding range can be easily limited by adding the position of the first emitter in the packet and discarding it if the forwarding node is more than $X \mathrm{~m}$ away from the first node. Moreover, a dissemination direction can also be defined by setting it in the message.

\section{Simulation SETUP}

In this section, we describe the mobility trace, the simulator and the metrics used in order to realize the performance evaluation of the schemes presented in the previous section.

\section{A. The Madrid trace}

The Madrid Highway trace [5] is a set of realistic micromobility traces for portions of two Madrid highways: the A6 and the M40. These traces give the position and speed of the nodes at each second during $1800 \mathrm{~s}$, over a $10 \mathrm{~km}$ 


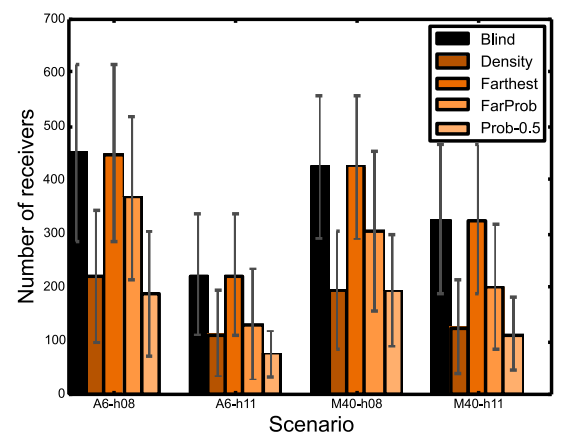

(a) Number of receivers of the packet

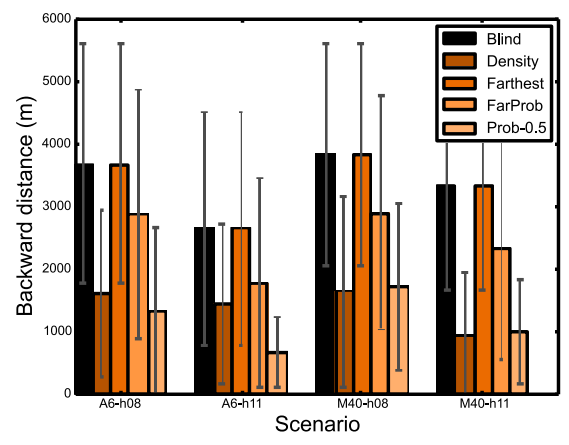

(c) Backward distance traveled by the packet

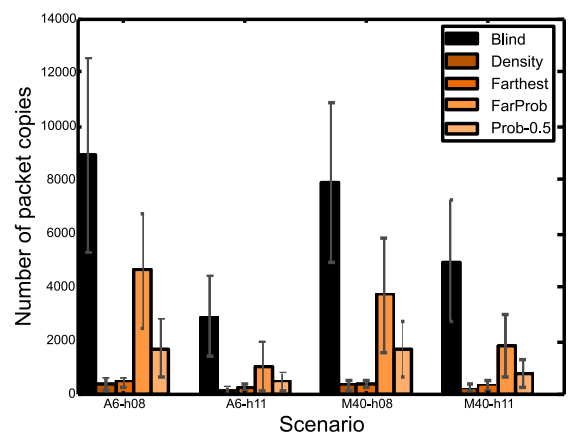

(b) Number of received packet copies

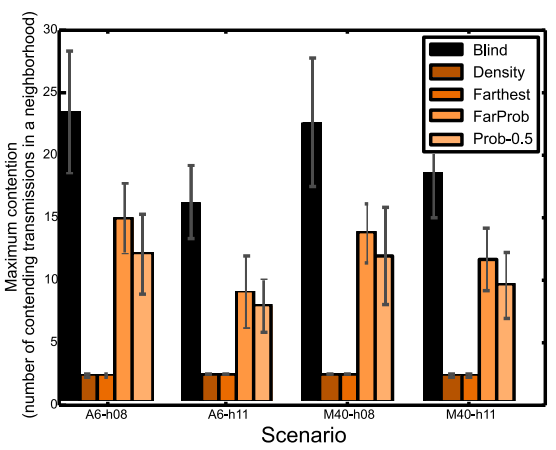

(d) Maximum number of contenders in a neighborhood

Fig. 1: General performance

portion of the highways, at different hours and days: Monday, Tuesday, Wednesday and Friday at 8h30 a.m. and 11h30 a.m. The traces are generated from traffic count data collected from induction loop sensors. The original dataset includes: the timestamp of the vehicle crossing the loop, the speed of the vehicle and the lane (both A6 and M40 have three lanes). From this original dataset, the trace itself is generated using a microscopic vehicular mobility simulator developed by the authors of [5] which is based on state of the art carfollowing and lane-changing models. The resulting trace is a text file in which each line presents the following information: [time $(\mathrm{s}), \operatorname{nodeID}, \mathrm{x}(\mathrm{m}), \mathrm{y}(\mathrm{m}), \operatorname{speed}(\mathrm{km} / \mathrm{h})]$.

\section{B. The simulator}

In order to evaluate the dissemination schemes on the Madrid traces, we developed our own lightweight simulator. The main reason why we did not use a classic discrete event simulator (NS2, Omnet++, etc) is that the number of nodes in the trace is too large to be handled by such simulators with the full network stack modeled. The tool we developed allows to evaluate the performance of the previously presented generic dissemination strategies on large scale traces. It performs the following tasks for each second of the trace:

1) parse the trace file and build its graph representation;

2) execute the algorithm on the graph: neighbors exchange messages according to the evaluated algorithm.

During the first step, the graph is a simple geometric graph where nodes are considered neighbors if they are separated by less than a given range. Concerning the results presented in this paper, the range is $150 \mathrm{~m}$, it is considered as a realistic value according to [5]. For the second step, one of the dissemination algorithm presented in Section III is executed on the graph. As the graph changes only every seconds and a message can be sent more rapidly with the standard MAC and physical layers [5], we choose to perform several steps of the algorithm on the same graph. In our case we choose to perform 5 steps of the algorithm during one second. For most of the presented algorithms, a step executed by a node consists in broadcasting a message to its neighbors. We thus consider that the MAC and Physical layers delays are of $200 \mathrm{~ms}$. When the algorithm converges (meaning that no more packets are scheduled to be sent), the simulator terminates. The outputs consist in several result metrics which are described in the next subsection.

In this paper, the considered simulation scenario is the following: at a given time, a node at the center of the highway portion is chosen to be the source of the information to disseminate. We choose dissemination start times as $i \times 200 \mathrm{~s}$ with $i \in 1, \ldots, 8$. In each simulation run presented in this paper, one source disseminates one information message.

\section{The considered metrics}

In order to compare the different protocols, we use the following metrics:

1) the number of receivers of the packet which allows to evaluate the delivery efficiency of the protocols;

2) the number of packet copies received which allows to indicate the level of redundancy of each protocol; 
3) the maximum contention which is the maximum number of contending packets in the neighborhood of a node (the maximum is taken over all the nodes and the duration of the simulation), and allows to evaluate how much the MAC layer is stressed;

4) the backward distance traveled by the packet which indicates the ability of the protocol to diffuse the information in space.

We compute forward distance as well but we only present backward distance in the paper as the results are very similar.

\section{RESULTS AND DISCUSSIONS}

In this section, we present and discuss the results obtained from the simulator. Two types of results are shown: first the metrics once the algorithms have converged, and then the temporal dynamics of the metrics until the algorithms converge. Finally we compare the results to the Carry-andForward version of Farthest Node dissemination to determine the usefulness to let the vehicles carry information.

\section{A. Memory-less algorithms performance}

In this section, we are interested only in memoryless algorithms (without Carry-and-Forward).

1) General Performance: The first thing we have to notice on the graphs of Fig. 1 is the large standard deviation bars. They account for the large variety of situations that can occur in the traces: when the packet is sent, depending whether the network is fully connected or on the contrary very sparse, the algorithms will behave very differently. As the results of [5] suggest, there is a large variability in the situations encountered over time, even for one single trace scenario. This variability of situations has to be taken care of by the dissemination protocols.

In Figs. 1a, 1b, 1c and 1d we monitor respectively the number of receivers, the number of received packet copies, the backward distance covered by the packet and the maximum contention for different scenarios and different schemes. We have to note that there are on average around 530 vehicles present on the simulated portion of the highway. First, we observe that $\mathrm{BF}$ and FF are the most efficient flooding algorithms in terms of number of receivers and distance covered by the packet, because they allow the packet to reach all the nodes of the connected component of the sender (as said, the variability in the results comes from the variability of the size of this connected component in the traces). Nevertheless, as can be observed on Figs. $1 \mathrm{~b}$ and $1 \mathrm{~d}, \mathrm{BF}$ induces more redundant receptions of the packet and the maximum contention is much higher. Although redundancy can be viewed as a good feature as it increases the reliability of the dissemination, it also causes congestion and collisions on the wireless medium. In the case of VANETs with a 802.11p MAC, high contention is really an issue [17]. FF seems thus preferable in the context of vehicular networks.

Then, the probabilistic schemes have medium performance with PFF being better than PF-0.5 (it corresponds to the PF scheme described in Section III with $p=0.5$ ): they are not as good as $\mathrm{BF}$ and $\mathrm{FF}$ in terms of number of received packets and covered distance, but induce less redundancy and maximum contention than BF. Regarding the PF scheme, we only consider $p=0.5$ here. Nevertheless, we can note that, if $p$ grows the PF scheme performance will converge toward the $\mathrm{BF}$ scheme performance.

Finally, concerning the density-based scheme DF, it performs not very well in terms of number of receivers and covered distance. This can be explained as follows: if the current forwarder (the highest degree neighbor of the previous forwarder) is close to the previous one, its neighbors may have already received the message from the previous forwarder, so the algorithm converges before flooding the entire connected component. In terms of redundancy, DF offers similar performances as FF.

These results thus show, as it was the case for previous studies with less realistic mobility setup [2], that FF is the best scheme for dissemination in vehicular networks. Interestingly, we observe that this result holds for all the 4 considered scenarios.

2) Timing Dynamics: In this section we compare the timing dynamics of the considered dissemination schemes. We are notably interested in the speed of the information dissemination which is a tremendously important parameter for road safety applications. We consider only the results for the A6h11 scenario because of the limited available space, but the results for other scenarios are qualitatively similar.

First, we observe on Fig. 2 that all the algorithms converge before 10 seconds. This delay can appear very large for safety applications. Nevertheless, as depicted in Fig. 1c, most of the schemes are able to cover a distance of 500 meters in less than one second, which might be good enough in function of the target application [6].

Figs. 2a and $2 \mathrm{c}$ are very similar as they depict respectively the number of receivers and the backward distance covered by the packet in function of time after its first emission. We observe in both cases, a linear growth followed by an inflection for all the schemes. We note that for the schemes with the best performance for these two metrics, the inflection is more delayed and thus the information flooded farther. Concerning the number of packet copies in Fig. 2b, the growth is similar as the number of receivers and backward distance, but the curves for the different schemes are separated earlier; FF and DF highly outperforming the other schemes. As for the maximum contention, concerning the BF, PF-0.5 and PFF the curves show a peak at the beginning of the dissemination because a lot of nodes have never received the packet and thus a lot of retransmissions happen, and then the number decreases with time. For the DF and FF schemes, there is a small peak of value 2 at the beginning as 2 neighbors of the first sender forward the packet (one for each direction) and then only one neighbor forwards it at each step.

We can conclude that during the first second (corresponding to 5 steps of the algorithm in our case), all the schemes have approximately the same performance in terms of dissemination (number of receivers and distance covered). Nevertheless, FF and DF induce less contention and on the long run FF outperforms DF for other metrics. 


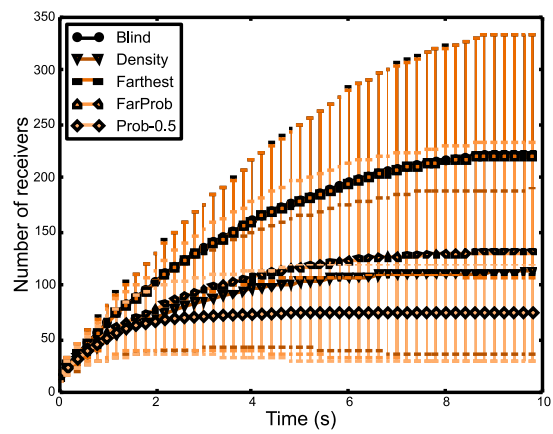

(a) Number of receivers of the packet

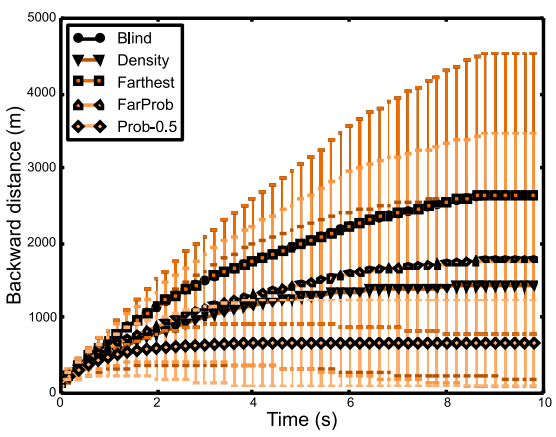

(c) Backward distance traveled by the packet

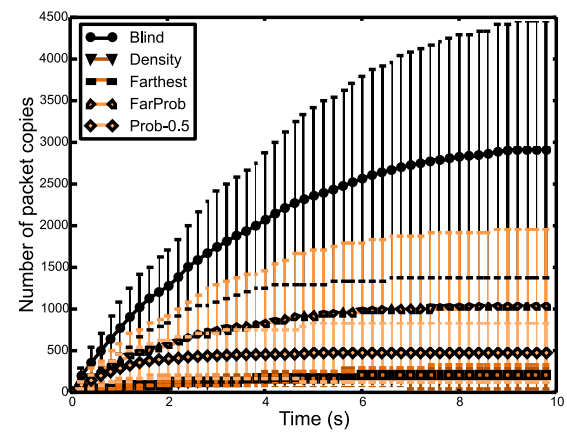

(b) Number of received packet copies

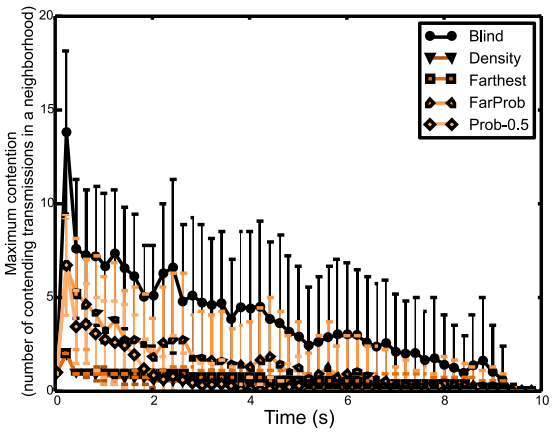

(d) Maximum number of contenders in a neighborhood

Fig. 2: Timing dynamics

\section{B. Is Carry-and-Forward relevant?}

In the previous subsection, we commented the performance of dissemination algorithms without memory. We concluded that FF is the best scheme as it allows to reach a maximum of nodes with a minimum of contention induced. In this subsection, we investigate the possibility to add carry-andforward capability to FF. The idea is to let nodes which have no neighbors farther in the dissemination direction to keep the packet until one appears. It notably allows nodes at the edges of a connected component to wait for components to merge and thus spread farther the information instead of letting the algorithm converge prematurely. In this case, the convergence of the algorithm can thus be decided with a delay since first emission or distance from first emitter criterion.

Fig. 3 depicts the comparison of performance of the carryand-forward version of FF with the non carry-and-forward schemes. We let run the simulation for 200 seconds in this case. Since the non carry-and-forward schemes converge before 10 seconds, their performance then remains constant. On Fig. $3 \mathrm{a}$ and $3 \mathrm{c}$, we observe that the carry-and-forward version of FF dissemination performance is at least as good as the best scheme of non carry-and-forward schemes during the first 10 seconds. After 10 seconds the number of receivers, and even more the covered distance, continue to increase. At first rapidly and then an inflection occurs as the dissemination packet reaches the edge of the simulated portion of the highway. After that, the packet is still disseminated to vehicles which continue to appear in the simulated trace. Concerning the number of packet copies which can be seen in Fig. 3b, for the first 10 seconds, it is similar to the FF and DF schemes as expected. Nevertheless, it then grows linearly with time. This is due to nodes at the edges of connected components which will receive the packet several times when new nodes are added to the component. It is especially the case with this trace, because nodes appear slowly at the beginning of the portion of the simulated highway. Thus every time a node appears, it is farther from the current carrier of the packet and it triggers a new transmission. Finally, on Fig. 3d, we can observe that the maximum contention remains very low when using the carryand-forward version of FF. Even in this configuration where cars appear one by one at the edge of the simulated portion of the highway, the number of packet copies and contention remains acceptable. We thus conclude that the addition of carry-and-forward capabilities to the FF scheme is desirable as it increases the performance of the scheme at a very low cost in terms of redundancy and contention.

\section{CONCLUSION AND FUtURE WORKS}

In this paper, we investigated the performance of several classic dissemination schemes. The performance evaluation is done in a realistic mobility context using the Madrid Highway trace. In order to make the simulations of the dissemination schemes on the traces scalable, we developed an adapted simulation tool. The results show that the FF scheme outperforms the others. Interestingly, the performance of the DF scheme which has been expected close to the FF is in fact much smaller. Finally we show the benefit of adding carry-andforward capabilities to the best performing scheme: FF. It allows to further improve its performance at a very marginal 


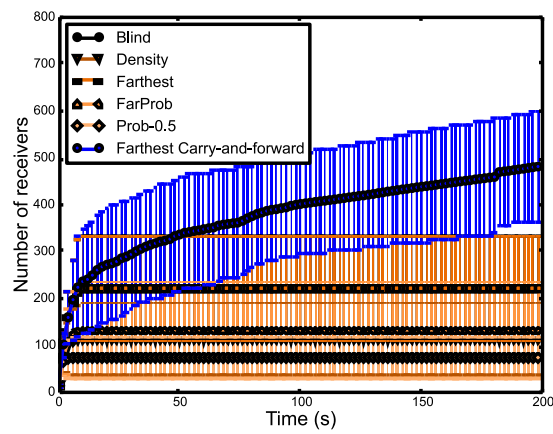

(a) Number of receivers of the packet

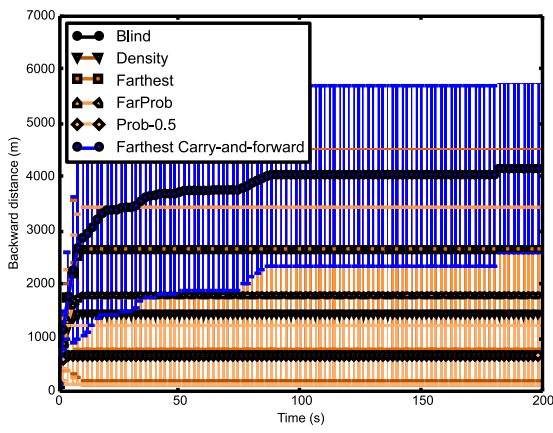

(c) Backward distance traveled by the packet

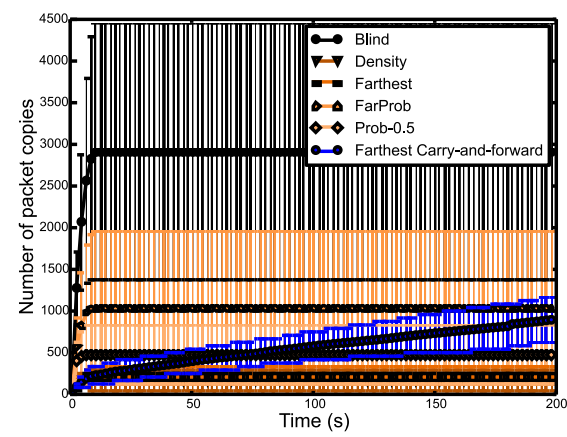

(b) Number of received packet copies

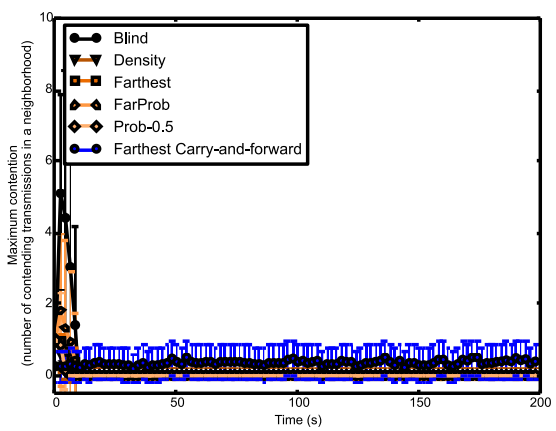

(d) Maximum number of contenders in a neighborhood

Fig. 3: Comparison with a carry-and-forward scheme

cost in terms of network load. In the future, we consider taking into account cars traveling in the opposite direction in the performance evaluation. We also plan to evaluate several carryand-forward approaches using FF in a more complex mobility environment such as urban mobility.

\section{ACKNOWLEDGMENT}

The authors would like to thanks Mohamed Omar Mazouz and Riadh Tahri for their role in the development of the simulator.

\section{REFERENCES}

[1] ETSI, "102 637-2: Intelligent Transport Systems (ITS)," Vehicular communications.

[2] A. T. Giang, A. Busson, and V. Vèque, "Message dissemination in vanet: protocols and performances," in Wireless Vehicular Networks for Car Collision Avoidance. Springer, 2013, pp. 71-96.

[3] M. Chaqfeh, A. Lakas, and I. Jawhar, "A survey on data dissemination in vehicular ad hoc networks," Vehicular Communications, vol. 1, no. 4, pp. 214-225, 2014.

[4] L. A. Villas, A. Boukerche, G. Maia, R. W. Pazzi, and A. A. Loureiro, "Drive: An efficient and robust data dissemination protocol for highway and urban vehicular ad hoc networks," Computer Networks, vol. 75, pp. 381-394, 2014.

[5] M. Gramaglia, O. Trullols-Cruces, D. Naboulsi, M. Fiore, and M. Calderon, "Vehicular networks on two madrid highways," in IEEE SECON, Singapore, 2014.

[6] H. Hartenstein and K. Laberteaux, VANET: vehicular applications and inter-networking technologies. Wiley Online Library, 2010.

[7] C.-F. Chiasserini, E. Fasolo, R. Furiato, R. Gaeta, M. Garetto, M. Gribaudo, M. Sereno, and Zanella, "Smart broadcast of warning messages in vehicular ad hoc networks," 2005.
[8] C.-C. Hung, H. Chan, and E. H.-K. Wu, "Mobility pattern aware routing for heterogeneous vehicular networks," in IEEE WCNC, Las Vegas, USA, 2008, pp. 2200-2205.

[9] S. Panichpapiboon and G. Ferrari, "Irresponsible forwarding," in IEEE ITST, Phuket, Thailand, 2008.

[10] R. S. Schwartz, R. R. Barbosa, N. Meratnia, G. Heijenk, and H. Scholten, "A directional data dissemination protocol for vehicular environments," Computer Communications, vol. 34, no. 17, pp. 20572071, 2011.

[11] H. Alshaer and E. Horlait, "An optimized adaptive broadcast scheme for inter-vehicle communication," in IEEE VTC Spring, Stockholm, Sweden, 2005.

[12] A. Wegener, H. Hellbrück, S. Fischer, C. Schmidt, and S. Fekete, "Autocast: An adaptive data dissemination protocol for traffic information systems," in IEEE VTC Fall, Baltimore, MD, USA, 2007.

[13] N. Wisitpongphan, O. Tonguz, J. S. Parikh, P. Mudalige, F. Bai, and V. Sadekar, "Broadcast storm mitigation techniques in vehicular ad hoc networks," Wireless Communications, IEEE, vol. 14, no. 6, pp. 84-94, 2007.

[14] Y.-T. Tseng, R.-H. Jan, C. Chen, C.-F. Wang, and H.-H. Li, "A vehicledensity-based forwarding scheme for emergency message broadcasts in vanets," in Mobile Adhoc and Sensor Systems (MASS), 2010 IEEE 7th International Conference on. IEEE, 2010, pp. 703-708.

[15] H. . F. Wu, R. . Guensler, R., and M. Hunter, "Mddv: a mobility-centric data dissemination algorithm for vehicular networks," in ACM VANET, 2004.

[16] J. Zhao and G. Cao, "Vadd: Vehicle-assisted data delivery in vehicular ad hoc networks," vol. 57, no. 3, pp. 1910-1922, May 2008.

[17] R. Stanica, E. Chaput, and A.-L. Beylot, "Broadcast communication in vehicular ad-hoc network safety applications," in IEEE CCNC, Las Vegas, USA, 2011. 